\title{
Conditions for a successful right-handed Majorana sneutrino curvaton
}

\author{
John McDonald \\ Dept. of Mathematical Sciences, University of Liverpool, Liverpool L69 3BX, United Kingdom
}

(Received 12 May 2004; published 16 September 2004)

\begin{abstract}
We consider the conditions which must be satisfied for a Majorana RH sneutrino, a massive righthanded (RH) sneutrino associated with the seesaw mechanism of Majorana neutrino masses, to play the role of the curvaton. Planck-scale suppressed nonrenormalizable terms in the RH neutrino superpotential must be eliminated to a high-order if the RH sneutrino curvaton is to dominate the energy density before it decays, which can be achieved via an R-symmetry which is broken to R-parity by the Giudice-Maseiro mechanism. In order to evade thermalization of the curvaton condensate, one $\mathrm{RH}$ neutrino mass eigenstate must have small Yukawa couplings, corresponding to a lightest neutrino mass $m_{\nu_{1}} \lesssim 10^{-3} \mathrm{eV}$. A time-dependent lepton asymmetry will be induced in the RH sneutrino condensate by the Affleck-Dine mechanism driven by SUSY breaking B-terms associated with the RH neutrino masses. Requiring that the resulting baryon asymmetry and isocurvature perturbations are acceptably small imposes an upper bound on the RH neutrino mass. We show that a scenario consistent with all constraints is obtained for a RH neutrino mass in the range $10^{2}-10^{4} \mathrm{GeV}$ when the RH sneutrino decay temperature is greater than $100 \mathrm{GeV}$ and lightest neutrino mass $m_{\nu_{1}} \approx 10^{-3} \mathrm{eV}$. Larger $\mathrm{RH}$ neutrino masses are possible for smaller $m_{\nu_{1}}$. The resulting scenario is generally consistent with a solution of the cosmic string problem of D-term inflation.
\end{abstract}

DOI: 10.1103/PhysRevD.70.063520

PACS numbers: $98.80 . \mathrm{Cq}$

\section{INTRODUCTION}

The possibility that the density perturbations responsible for structure formation come from quantum fluctuations of a scalar field other than the inflaton has recently attracted considerable interest [1-4]. The scalar field, known as the curvaton [3], is effectively massless during inflation, but begins coherent oscillations after inflation and comes to dominate the energy density of the Universe before it decays. The initial isocurvature perturbations of the curvaton are then converted to adiabatic energy density perturbations. A number of curvaton candidates have been proposed and analyses of curvaton dynamics performed [4-10].

An important question is whether there is a natural curvaton candidate in the context of the minimal supersymmetric (SUSY) Standard Model (MSSM) and its extensions. SUSY is advantageous as it allows us to understand the flatness of the curvaton potential with respect to quantum corrections. A specific motivation for a SUSY curvaton is provided by D-term hybrid inflation [11]. In D-term inflation with naturally large values of the superpotential coupling, the energy density during inflation must be lowered in order to evade the problem of cosmic microwave background (CMB) perturbations due to cosmic strings [12]. This, in turn, requires an alternative source of the cosmological energy density perturbations, such as a curvaton.

Some general conditions for a SUSY flat direction scalar to play the role of a curvaton were considered in [6]. Similar conditions for the specific case of right-

*Email: mcdonald@amtp.liv.ac.uk handed (RH) sneutrinos (the scalar superpartners of the $\mathrm{RH}$ neutrinos) were discussed in [7]. It was shown that small couplings of the curvaton to the MSSM fields are required in order to evade thermalization of the curvaton condensate by scattering from particles in the thermal background and in order to have a low enough decay temperature for the curvaton condensate to dominate the energy density before it decays. Curvaton domination also requires the elimination of Planck-scale suppressed nonrenormalizable terms (NRT) to a high order in the flat direction superpotential.

The case of a coherently oscillating MSSM flat direction condensate formed from a linear combination of squark, slepton and Higgs fields was considered in [8]. It was shown that, as a result of their interaction with the fields in the background radiation, the condensates are either thermalized or fail to dominate the energy density before they decay. MSSM flat direction scalars can therefore play the role of a curvaton only if one assumes that the inflaton can primarily decay to "hidden radiation" consisting of hypothetical light hidden sector fields [8]. In the following we will consider the conventional case of inflaton decay to observable radiation.

An alternative curvaton candidate, in the context of the MSSM extended to accommodate neutrino masses, is a RH sneutrino. The curvaton condensate is most likely to survive in the thermal environment of the early Universe and to dominate the energy density before it decays if it is relatively weakly coupled to MSSM fields. This makes a RH sneutrino a promising candidate for a SUSY curvaton. This possibility was noted in [6] and discussed in [2,13], which also considered the possibility of leptogenesis via RH sneutrino decay and the associated isocurvature 
fluctuations. In [7] the conditions for a RH sneutrino to serve as a curvaton were considered in some detail.

The results of [7] showed that a Dirac RH sneutrino (a RH sneutrino associated with Dirac neutrino masses, corresponding to RH neutrino masses $M_{N}=0$ ) could serve as a curvaton if the amplitude of the RH sneutrino at the onset of its coherent oscillations satisfied $N_{\text {osc }} \geqslant$ $10^{-5} M$, where $M=M_{P l} / \sqrt{8 \pi}$ with $M_{P l}$ the Planck mass. This requires that Planck-scale suppressed superpotential NRT $\propto N^{n}$ are suppressed for $n<6$ [7]. The weak Yukawa coupling of the Dirac RH sneutrino ensures that there is no danger of condensate thermalization. However, the decay temperature of the RH sneutrino condensate was found to be around $10 \mathrm{MeV}$, which introduces two problems [7]. The decay of the condensate RH sneutrinos will produce a large number of lightest SUSY particles (LSPs), which will overclose the Universe unless they decay sufficiently rapidly. In order that their decay products do not destroy the light elements produced in nucleosynthesis, the RH sneutrinos and LSPs must decay before nucleosynthesis occurs at $T_{\text {nuc }} \approx 1 \mathrm{MeV}$. This requires new R-parity-violating interactions in order to have sufficiently rapid LSP decay. (This also has the disadvantage of eliminating the possibility of LSP dark matter.) The second problem is that the baryon asymmetry must be produced during the decay or after the decay of the condensate RH sneutrinos, since any preexisting baryon asymmetry will not be correlated with the entropy fluctuations produced by curvaton decay, resulting in unacceptably large baryon isocurvature fluctuations [5]. Therefore, new baryon-number-violating interactions must be introduced in order that the RH sneutrino decay can directly produce the baryon asymmetry. While no definitive analysis of these possibilities has yet been undertaken, the Dirac RH sneutrino curvaton appears to be disfavored.

Majorana RH sneutrinos ( $\mathrm{RH}$ sneutrinos associated with the seesaw mechanism for Majorana neutrino masses [14], corresponding to the case $M_{N} \neq 0$ ) can also dominate the energy density of the Universe before they decay. However, because of the larger Yukawa couplings in the seesaw mechanism for neutrino masses, the RH sneutrino condensate decays earlier, requiring a larger initial amplitude, $N_{\text {osc }} \gtrsim 10^{-3} M$, and correspondingly greater suppression of the Planck-suppressed superpotential NRT (all superpotential terms $\propto N^{n}$ with $n<11$ [7]). In addition, the Majorana RH sneutrino curvaton was shown to have a potential problem with condensate thermalization. Because of its larger Yukawa couplings, the interaction of the Majorana RH sneutrino curvaton condensate with the MSSM fields in the thermal background is much stronger than in the case of the Dirac RH sneutrino. As a result, any contribution to the effective light neutrino mass matrix resulting from couplings to the curvaton's fermionic partner must be less than
$10^{-3} \mathrm{eV}$ in order to avoid thermalization of the condensate [7]. Thus, if the masses of the neutrinos are characterized by the mass squared splittings suggested by solar and atmospheric neutrino oscillations, $m_{\nu} \sim 0.01-$ $0.1 \mathrm{eV}$ [15], the RH sneutrino condensate would be thermalized before it could dominate the energy density. This will be the case if there is no correlation between the $\mathrm{RH}$ neutrino mass matrix and the neutrino Yukawa coupling matrix, in the sense that diagonalization of the RH neutrino mass matrix does not result in approximate diagonalization of the neutrino Yukawa coupling matrix. In this case the RH sneutrino curvaton mass eigenstate would have Yukawa couplings to the Higgs and lepton doublets characterized by the largest neutrino mass eigenvalue, $m_{\nu} \approx 0.1 \mathrm{eV}$, ruling out the Majorana $\mathrm{RH}$ sneutrino as a curvaton.

However, it is possible for mass squared splittings and mixing angles associated with solar and atmospheric neutrino phenomenology to be accounted for by just two RH neutrino mass eigenstates. For example, this is the case in the single right-handed neutrino dominance (SRHND) model of neutrino masses [16]. A third righthanded neutrino and its superpartner could then have arbitrarily small Yukawa couplings to the MSSM fields, allowing its condensate to evade thermalization. In this case the Majorana RH sneutrino becomes a viable curvaton candidate. The requirement that the lightest neutrino mass satisfies $m_{\nu_{1}} \lesssim 10^{-3} \mathrm{eV}$ may then be regarded as a prediction of the Majorana RH sneutrino curvaton model.

In this paper we consider in detail the case of a Majorana RH sneutrino curvaton with a light neutrino mass eigenstate, which was not considered in [7]. An advantage of the Majorana RH sneutrino curvaton with respect to dangerous Planck-suppressed NRT is that the conventional R-symmetry of the MSSM (broken to Rparity by the $\mu H_{u} H_{d}$ term) [17], extended to include the RH neutrinos, will completely eliminate all RH neutrino superpotential terms except the mass term and Yukawa coupling terms responsible for neutrino masses, thus solving the problem of dangerous Planck-suppressed NRT. The R-symmetry must be broken to R-parity, but if this occurs via the Giudice-Masiero mechanism [18], which generates only R-symmetry breaking effective superpotential terms with total R-charge equal to zero, then no dangerous RH neutrino superpotential NRT will arise. Operators eliminated by a global symmetry such as R-symmetry might be generated by quantum gravity wormhole effects [19]. However, this is dependent upon the short-distance structure of gravity [20]. In the following we will take the view that quantum gravity corrections are absent or highly suppressed.

We will show that a time-dependent lepton asymmetry is induced in the RH sneutrino condensate via a variant of the Affleck-Dine (AD) mechanism driven by the SUSY breaking B-terms associated with the $\mathrm{RH}$ neutrino 
masses. (This mechanism has also recently been discussed in the more general context of AD leptogenesis via a RH sneutrino condensate in [21].) Unlike the conventional AD mechanism associated with MSSM flat direction fields [22], L-violation from the B-terms remains unsuppressed throughout the evolution of the condensate, resulting in a rapidly oscillating lepton asymmetry. If the RH sneutrino decay occurs before the electroweak phase transition, as is likely to be necessary in order to generate the baryon asymmetry, then any lepton asymmetry will be processed into a baryon asymmetry of the same order of magnitude by sphalerons, which must be less than the observed baryon asymmetry of the Universe. The requirement that the lepton number to entropy ratio due to the decay of the RH sneutrino condensate satisfies $n_{L} / s \lessgtr 10^{-10}$ will be shown to impose a significant constraint on the Majorana RH sneutrino curvaton scenario. In particular, it will be shown that $\mathrm{RH}$ sneutrino leptogenesis via $C P$-violating decays of the condensate RH sneutrinos [13] can account for the baryon asymmetry only if the lightest neutrino mass is less than about $10^{-8} \mathrm{eV}$. This favors electroweak baryogenesis [23] as the origin of the baryon asymmetry in this scenario.

The paper is organized as follows. In Section II we discuss the conditions for a Majorana RH sneutrino to dominate the energy density and to account for the observed energy density perturbations. We also discuss the possibility that a Majorana RH sneutrino could account for the energy density perturbations in D-term inflation. In Section III we discuss the constraints on the RH neutrino Yukawa couplings and neutrino masses coming from evasion of condensate thermalization. We also discuss the possibility that thermal effective masses could play a role in the evolution of the condensate. In Section IV we consider the AD lepton asymmetry induced in the Majorana RH sneutrino condensate by the B-term AD mechanism and the associated constraints on the model. In Section V we discuss the range of model parameters allowed by the constraints. In Section VI we present our conclusions.

\section{CURVATON DOMINANCE AND DENSITY PERTURBATION CONSTRAINTS}

We consider the simplest inflation scenario, in which the expansion rate during inflation, $H_{I}$, is constant and inflation is followed by a period of coherent inflaton oscillations. (We refer to this period as inflaton matter domination, IMD). Inflaton oscillations decay away completely at the reheating temperature, $T_{R}$, corresponding to the temperature at which the Universe becomes radiation dominated.

We will consider throughout the case where the reheating temperature from inflaton decay satisfies the thermal gravitino constraint [24], $T_{R} \lesssim 10^{8} \mathrm{GeV}$ for $m_{3 / 2}=$
$100 \mathrm{GeV}\left(T_{R} \lesssim 10^{9} \mathrm{GeV}\right.$ for $\left.m_{3 / 2}=1 \mathrm{TeV}\right)$ [25]; in the following we will consider the constraint for $m_{3 / 2}=$ $100 \mathrm{GeV}$. This constraint is not necessary if the curvaton dominates the energy density for long enough that it dilutes the thermal gravitinos due to inflaton reheating. However, we will be considering the limiting case where the curvaton just dominates the energy density before it decays, so we will impose the thermal gravitino constraint for consistency. In this case the expansion rate at the end of IMD will satisfy $H\left(T_{R}\right) \lesssim 10^{-2} \mathrm{GeV}(1 \mathrm{GeV})$ for $T_{R} \lesssim 10^{8} \mathrm{GeV}\left(10^{9} \mathrm{GeV}\right)$, which is small compared with the value $H_{\mathrm{osc}} \approx m_{N} \geqslant m_{s} \approx 100 \mathrm{GeV}$ at which $\mathrm{RH}$ sneutrino coherent oscillations begin. Here $m_{N}$ is the RH sneutrino mass, $m_{s}$ is the gravity-mediated soft SUSY breaking scalar mass and $H\left(T_{R}\right)=k_{T} T_{R}^{2} / M$ is the expansion rate at the onset of radiation domination, where $k_{T}=$ $\left[\pi^{2} g(T) / 90\right]^{1 / 2}$ and $g(T)$ is the number of degrees of freedom in thermal equilibrium at $T$ [26]. (In the following we will consider $g(T) \approx 200$, corresponding to all MSSM fields in equilibrium, such that $k_{T} \approx 4$.7.) Thus we will consider the RH sneutrino oscillations to begin during IMD.

The superpotential of the mass eigenstate RH neutrinos, $N_{i}$, is given by

$$
W_{\nu}=\lambda_{\nu i j} N_{i} H_{u} L_{j}+\frac{M_{N_{i}} N_{i}^{2}}{2},
$$

where $H_{u}$ and $L_{i}$ are the MSSM Higgs and lepton doublets, respectively, and $i$ is a generation index. This leads to Majorana neutrino masses via the seesaw mechanism [14]. In addition, we generally expect Planck-scale suppressed nonrenormalizable superpotential terms involving the $\mathrm{RH}$ neutrino superfields of the form

$$
W=\frac{\lambda_{n} N_{i}^{n}}{n ! M^{n-3}} .
$$

In [7] it was shown that such terms must be eliminated for all $n<11$ if the initial amplitude of the Majorana $\mathrm{RH}$ sneutrino curvaton is to be large enough for the coherently oscillating curvaton to dominate the energy density before it decays. Otherwise the contribution of Plancksuppressed terms to the effective mass squared of the RH sneutrino will be greater than $H^{2}$, thus driving the RH sneutrino amplitude to small values. This elimination of the Planck-suppressed NRT requires an explanation in terms of a symmetry or other mechanism. One possibility is an R-symmetry. The superpotential Eq. (1) is Rsymmetric under a simple extension of the R-symmetry of the MSSM under which $R\left(Q, L, u^{c}, d^{c}, e^{c}\right)=1$ and $R\left(H_{u}, H_{d}\right)=0$ [17]. By assigning $R(N)=1$, all the terms in Eq. (1) are permitted by the R-symmetry while all superpotential terms in Eq. (2) with $n \neq 2$ are excluded. The $\mu H_{u} H_{d}$ term, essential for satisfactory electroweak symmetry breaking [17], breaks this R-symmetry to Rparity. The Giudice-Masiero mechanism [18] initially 
eliminates the $\mu$-term via an R-symmetry. The $\mu$-term is then generated by R-symmetry breaking due to a nonminimal kinetic term in the Kähler potential of the form $z^{\dagger} H_{u} H_{d}$, coupling the SUSY breaking hidden sector superfield $z$ to the Higgs doublets. Since $R(z)=0$, this term is permitted by the R-symmetry. The F-term of the $z$ superfield breaks both SUSY and the R-symmetry, resulting in a $\mu$-term of the order of the electroweak scale in the superpotential of the low-energy effective theory [18]. The important feature of this mechanism is that it only generates R-symmetry breaking terms in the low-energy effective superpotential which have total R-charge equal to zero. As a result no superpotential terms of the form $N^{n}$ $(n \neq 2)$ will arise. However, this mechanism does require that some interactions between the hidden sector and observable sector superfields are eliminated by a mechanism other than R-symmetry, since R-symmetry permits a superpotential term of the form $z N^{2}$, which would typically lead to a very large RH neutrino mass due to the $z$ expectation value $\approx M$.

The scalar potential of the mass eigenstate RH sneutrinos after inflation is given by

$$
V(N)=\left(\frac{m_{N}^{2}-c H^{2}}{2}\right)|N|^{2}+B\left(\frac{M_{N} N^{2}}{2}+\text { h.c. }\right),
$$

where we have suppressed the generation indices. Here $m_{N}^{2}=m_{s}^{2}+M_{N}^{2}+m_{\mathrm{eff}}^{2}$, where $m_{\mathrm{eff}}^{2}=\lambda_{\nu}^{2}\left\langle\phi^{2}\right\rangle$ is the effective mass squared term due to the interaction of the $\mathrm{RH}$ sneutrino with thermal background MSSM fields, $\phi$ [27]. The latter will be shown to play no role in the evolution of the RH sneutrino curvaton. Since there is little motivation to study RH neutrino masses less than the order of $100 \mathrm{GeV}$, for simplicity we will restrict attention to the case $M_{N} \gtrsim m_{s}$ in the following and therefore consider $m_{N} \approx M_{N}$ throughout. In fact, we will show that the allowed values of $M_{N}$ are generally larger than $\mathrm{O}(100) \mathrm{GeV}$ in models where the curvaton condensate decays before the electroweak phase transition, which are the most promising with respect to baryogenesis.

The mass squared terms and possibly also B-terms gain corrections during IMD due to nonzero inflaton F-terms [28-30]. (During inflation such terms must be effectively zero in order to have a scale-invariant density perturbation spectrum, which is natural in D-term inflation [11].) We will consider a negative order $H^{2}$ correction after inflation with $c \approx 1$. This is because the evolution of the RH sneutrino under the influence of a positive mass squared term in the case $c \approx-1$ will suppress the initial amplitude of the RH sneutrino oscillation relative to its amplitude at the end of inflation. As we show later, this would require a large value of $N$ during inflation in order for the curvaton to dominate the energy density before it decays, which would in turn require a large value of $H_{I}$ in order to have sufficiently large curvaton energy density perturbations. However, this large value of $H_{I}$ will gen- erally result in excessively large inflaton energy density perturbations and gravitational waves.

The RH sneutrino oscillations begin at $H_{\mathrm{osc}} \approx$ $M_{N} / c^{1 / 2} \approx M_{N}$. The energy density of the RH sneutrino condensate at $a>a_{R}$ is then [7]

$$
\frac{\rho_{N}}{\rho_{r}}=\left(\frac{a}{a_{R}}\right)\left(\frac{\rho_{N}}{\rho}\right)_{\mathrm{osc}}=\left(\frac{a}{a_{R}}\right) \frac{N_{\mathrm{osc}}^{2}}{6 M^{2}},
$$

where $a_{R}$ is the scale factor at the onset of radiation domination, $\rho_{r}$ is the radiation energy density, $\rho \approx$ $3 H^{2} M^{2}$ is the energy density due to the coherently oscillating inflaton field during IMD and $\rho_{N}=M_{N}^{2} N_{\text {osc }}^{2} / 2$ is the initial energy density in the RH sneutrino oscillations. RH sneutrino domination therefore occurs at [7]

$$
H_{\mathrm{dom}}=\left(\frac{N_{\mathrm{osc}}^{2}}{24 M^{2}}\right)^{2} H_{R}
$$

where $H \propto a^{-3 / 2}$ during IMD. In this expression we have defined curvaton domination to correspond to $\rho_{N}>4 \rho_{r}$, in order to ensure complete domination of the energy density by the curvaton.

We consider first the case of a single neutrino generation, which will later correspond to the RH sneutrino mass eigenstate with the weakest Yukawa couplings in Eq. (1). The RH sneutrino decay rate is given by

$$
\Gamma_{d} \approx \frac{\lambda_{\nu}^{2} M_{N}}{4 \pi}=\frac{m_{\nu} M_{N}^{2}}{4 \pi v^{2}},
$$

where the neutrino mass is given by the seesaw expression

$$
m_{\nu}=\frac{\lambda_{\nu}^{2} v^{2}}{M_{N}}
$$

and $\left\langle H_{u}\right\rangle=v / 2$. The decay temperature of the RH sneutrino condensate is then

$$
T_{d} \approx\left(\frac{\lambda_{\nu}^{2} M_{N} M}{4 \pi k_{T}}\right)^{1 / 2} \equiv\left(\frac{m_{\nu} M_{N}^{2} M}{4 \pi k_{T} v^{2}}\right)^{1 / 2} .
$$

Therefore

$$
\begin{aligned}
M_{N} \approx & 150\left(\frac{g\left(T_{d}\right)}{200}\right)^{1 / 4}\left(\frac{T_{d}}{100 \mathrm{GeV}}\right)\left(\frac{10^{-4} \mathrm{eV}}{m_{\nu}}\right)^{1 / 2} \\
& \times\left(\frac{v}{100 \mathrm{GeV}}\right) \mathrm{GeV} .
\end{aligned}
$$

From Eq. (9) we see that if $M_{N} \gtrsim 100 \mathrm{GeV}$ and $m_{\nu}$ is not much smaller than $10^{-4} \mathrm{eV}$ then $T_{d} \gtrsim 100 \mathrm{GeV}$. In this case there is a possibility of generating the baryon asymmetry either via electroweak baryogenesis [23] or via leptogenesis by RH sneutrino decay [13].

The condition for the RH sneutrino condensate to dominate the energy density before it decays, $H_{\text {dom }}>$ $\Gamma_{d}$, is given by

$$
\frac{N_{\text {osc }}}{M}>2\left(\frac{9}{\pi k_{T}}\right)^{1 / 4} m_{\nu}^{1 / 4} M^{1 / 4}\left(\frac{M_{N}}{T_{R} v}\right)^{1 / 2} .
$$


Therefore

$$
\begin{aligned}
\frac{N_{\mathrm{osc}}}{M} & >4.0 \times 10^{-3} \beta \\
\beta & =\left(\frac{m_{\nu}}{10^{-4} \mathrm{eV}}\right)^{1 / 4}\left(\frac{10^{8} \mathrm{GeV}}{T_{R}}\right)^{1 / 2}\left(\frac{M_{N}}{v}\right)^{1 / 2} .
\end{aligned}
$$

Thus if the mass of the lightest neutrino is not very much smaller than $10^{-4} \mathrm{eV}$ then $N_{\text {osc }}$ cannot be very much smaller than $M$.

A value of $N$ larger than $M$ implies that $\rho_{N} \approx$ $-H^{2} N^{2} \approx-\rho$, leading to a breakdown of the assumption that the energy density is dominated by inflaton oscillations after inflation. In addition, chaotic initial conditions with all initial gradient energy densities $\lesssim M^{4}$ would imply that no field can have a value at the onset of inflation significantly larger than $M$ [9]. We will therefore require that $N_{\text {osc }} \lesssim M$. From Eq. (11) a range of $N_{\text {osc }}$ compatible with curvaton domination then exists only if

$$
M_{N} \lesssim 0.06\left(\frac{10^{-4} \mathrm{eV}}{m_{\nu}}\right)^{1 / 2}\left(\frac{v}{100 \mathrm{GeV}}\right) T_{R}
$$

A second condition that the RH sneutrino curvaton must satisfy is that the curvaton energy density perturbations are of the correct magnitude to account for the CMB temperature fluctuations. The curvaton energy density perturbation is given by [3]

$$
\delta_{\rho}=\frac{2 \delta N}{N} \approx\left(\frac{H_{I}}{\pi N_{I}}\right),
$$

where $N_{I}$ is the amplitude of $N$ during inflation and $\delta N \approx$ $H_{I} / 2 \pi$ is the quantum fluctuation of the RH sneutrino. The observed perturbation is given by ${ }^{1} \delta_{\rho} \approx 1.4 \times 10^{-4}$ [3]. If $N$ does not evolve from the end of inflation then $N_{I} \approx N_{\text {osc }} \geqslant 10^{-3} M$, which would imply that $H_{I} \approx$ $\pi \delta_{\rho} N_{\text {osc }} \gtrsim 10^{12} \mathrm{GeV}$. However, it was observed in [7] that the RH sneutrino field will roll to larger values if the expected negative order $H^{2}$ mass squared correction arises after the end of inflation. In this case $N_{o s c} \gg N_{I}$ is possible, allowing smaller values of $H_{I}$ to be compatible with a RH sneutrino curvaton. During IMD, $H \propto$ $a^{-3 / 2}$. In this case the evolution of the RH sneutrino due to a $-c H^{2}$ mass squared term is given by $N \propto a^{\gamma}$, where [7]

$$
\gamma=\frac{1}{2}\left[-\frac{3}{2}+\sqrt{\frac{9}{4}+4 c}\right]
$$

Thus

$$
\frac{N_{\mathrm{osc}}}{N_{I}}=\left(\frac{a_{\mathrm{osc}}}{a_{e}}\right)^{\gamma}=\left(\frac{H_{I}}{H_{\mathrm{osc}}}\right)^{2 \gamma / 3},
$$

\footnotetext{
${ }^{1}$ More precisely, the power spectrum of the curvaton fluctuation is $\mathcal{P}_{\delta N / N} \equiv H_{I} / 2 \pi N_{I}=3 \mathcal{P}_{\zeta}^{1 / 2} / 2$, where the observed value of curvature spectrum is $\mathcal{P}_{\zeta}^{1 / 2 \zeta}=4.8 \times 10^{-5}$ [3].
}

where $a_{\mathrm{osc}}, a_{e}$ are the scale factors at the onset of curvaton oscillations and the end of inflation, respectively. The amplitudes of $N$ and $\delta N$ due to a mass squared correction evolve in the same way, therefore $\delta N / N$ remains constant and Eq. (13) is unaltered [7]. The curvaton domination condition Eq. (11) combined with Eq. (13) and (15) then implies that the lower bound on $H_{I}$ is given by

$$
H_{I}^{1+2 \gamma / 3} \geq 4.0 \times 10^{-3} \beta \pi \delta_{\rho} H_{\mathrm{osc}}^{2 \gamma / 3} M .
$$

The lower bound depends on $c$. If $c=1$, for example, then $\gamma=0.5$, and with $\delta_{\rho}=1.4 \times 10^{-4}$, the lower bound on the expansion rate during inflation becomes

$$
\begin{aligned}
H_{I} \geqslant & 9.3 \times 10^{9}\left(\frac{m_{\nu}}{10^{-4} \mathrm{eV}}\right)^{3 / 16}\left(\frac{10^{8} \mathrm{GeV}}{T_{R}}\right)^{3 / 8} \\
& \times\left(\frac{M_{N}}{100 \mathrm{GeV}}\right)^{5 / 8}\left(\frac{100 \mathrm{GeV}}{v}\right)^{3 / 8} \mathrm{GeV} .
\end{aligned}
$$

Smaller values of $H_{I}$ are possible with larger values of $c$. For example, with $c=2.5$, corresponding to $\gamma=1$, the lower bound becomes

$$
\begin{aligned}
H_{I} \gtrsim & 2.4 \times 10^{8}\left(\frac{m_{\nu}}{10^{-4} \mathrm{eV}}\right)^{3 / 20}\left(\frac{10^{8} \mathrm{GeV}}{T_{R}}\right)^{3 / 10} \\
& \times\left(\frac{M_{N}}{100 \mathrm{GeV}}\right)^{7 / 10}\left(\frac{100 \mathrm{GeV}}{v}\right)^{3 / 10} \mathrm{GeV} .
\end{aligned}
$$

For the case of a positive $H^{2}$ correction to the $\mathrm{RH}$ sneutrino mass squared with $c \approx-1$, we see from Eq. (14) that $\operatorname{Re}(\gamma)=-3 / 4$ and so $N_{\text {osc }} / N_{I}=$ $\left(H_{\text {osc }} / H_{I}\right)^{1 / 2} \approx\left(M_{N} / H_{I}\right)^{1 / 2}$. With $N_{I}$ related to $H_{I}$ by Eq. (13), $H_{I}$ is therefore given by

$$
H_{I}=\frac{\pi^{2} \delta_{\rho}^{2} N_{\mathrm{osc}}^{2}}{M_{N}}
$$

The lower bound on $N_{\text {osc }}$, Eq. (11), then implies that

$$
H_{I} \gtrsim 1.8 \times 10^{23}\left(\frac{m_{\nu}}{10^{-4} \mathrm{eV}}\right)^{1 / 2}\left(\frac{10^{8} \mathrm{GeV}}{T_{R}}\right) .
$$

$H_{I}$ is therefore much larger than the upper bound from gravitational waves produced during inflation, $H_{I} \lesssim$ $10^{-5} M[26]$.

One important application of a SUSY curvaton is to D-term hybrid inflation [11]. It has been shown that the contribution of $U(1)_{F I}$ strings to the CMB, assuming naturally large values of the order of 1 for the superpotential coupling, is inconsistent with observations in the case where the energy density perturbations are explained by conventional inflaton perturbations [12]. In order to evade this problem, the expansion rate during inflation must satisfy $H_{I} \lesssim 4 \times 10^{12} \mathrm{~g} \mathrm{GeV}$ [12], where $g$ is the Fayet-Iliopoulos gauge coupling [11]. In this case a new source of energy density perturbations is required, since conventional inflaton energy density perturbations 
are too small. From Eq. (17), requiring that $H_{I} \lesssim 4 \times$ $10^{12} \mathrm{~g} \mathrm{GeV}$ implies that a $\mathrm{RH}$ sneutrino curvaton with $c=1$ can evade the D-term inflation cosmic string problem and provide the energy density perturbations if

$$
\begin{aligned}
M_{N} \lesssim & 1.6 \times 10^{6} g^{8 / 5}\left(\frac{10^{-4} \mathrm{eV}}{m_{\nu}}\right)^{3 / 10}\left(\frac{T_{R}}{10^{8} \mathrm{GeV}}\right)^{3 / 5} \\
& \times\left(\frac{v}{100 \mathrm{GeV}}\right)^{3 / 5} \mathrm{GeV},
\end{aligned}
$$

while for $c=2.5$,

$$
\begin{aligned}
M_{N} \lesssim & 1.0 \times 10^{8} g^{10 / 7}\left(\frac{10^{-4} \mathrm{eV}}{m_{\nu}}\right)^{3 / 14}\left(\frac{T_{R}}{10^{8} \mathrm{GeV}}\right)^{3 / 7} \\
& \times\left(\frac{v}{100 \mathrm{GeV}}\right)^{3 / 7} \mathrm{GeV} .
\end{aligned}
$$

\section{THERMAL CONSTRAINTS ON MAJORANA RIGHT-HANDED SNEUTRINO CURVATONS}

In this section we discuss the constraints coming from the requirement that the Majorana $\mathrm{RH}$ sneutrino condensate is not thermalized before the condensate decays. We also comment on the possibility that an effective RH sneutrino mass due to the interaction of the curvaton RH sneutrino with the thermal background could play a role in the evolution of the condensate [27].

In [7] it was observed that a Majorana RH sneutrino condensate will be thermalized before it decays if the Yukawa couplings of the mass eigenstate RH neutrinos are all of the same order of magnitude. This would be expected if there was no correlation between the RH neutrino mass matrix and the Yukawa coupling matrix $\lambda_{\nu i j}$, in the sense that the basis in which the RH neutrino mass matrix is diagonalized is unrelated to the basis in which the Yukawa coupling matrix is diagonalized, since in this case all the mass eigenstate RH neutrino superfields would have similar Yukawa couplings to the lepton doublets. The thermalization rate would then be determined by the largest Yukawa coupling, corresponding to the neutrino mass indicated by atmospheric solar neutrino oscillations, $m_{\nu} \approx 0.1 \mathrm{eV}$.

However, it is possible for one of the RH neutrino mass eigenstates to have much weaker Yukawa couplings to the MSSM fields than those which are responsible for the mass splittings and mixing angles observed in solar and atmospheric neutrino oscillations [16]. In this case there will be a hierarchical light neutrino mass spectrum $m_{\nu_{1}} \ll m_{\nu_{2}} \ll m_{\nu_{3}}$, where $m_{\nu_{i}}(i=1,2,3)$ are the light Majorana neutrino mass eigenvalues [15]. It is then possible for coherent oscillations of the weakly coupled RH sneutrino to evade thermalization. Since coherent oscillations of the more strongly coupled RH sneutrinos will be thermalized by scattering from the thermal background MSSM fields, the weakly coupled RH sneutrino will naturally play the role of the curvaton.
For simplicity, we will consider the Yukawa couplings of the weakly coupled RH sneutrino mass eigenstate $N_{1}$ to lepton doublets $L_{i}$ to be all of the same order of magnitude, $\lambda_{\nu 1 i} \sim \lambda_{\nu}$. $\lambda_{\nu}$ will then determine the lightest neutrino mass $m_{\nu_{1}}$ via the seesaw mechanism with $N_{1}$. It will also determine the thermalization rate of the $\mathrm{RH}$ sneutrino condensate. We will drop the generation subscript and write $N_{1}$ as $N$ in the following.

The condition for the RH sneutrino condensate to evade thermalization is that the scattering rate of the most strongly coupled thermal background MSSM fields from the zero-momentum condensate $\mathrm{RH}$ sneutrinos is less than the expansion rate $H$. The strongest scatterings will be from top quarks/squarks in the thermal background via Higgs exchange. The scattering cross-section is given by [7]

$$
\sigma_{\mathrm{sc}} \approx \frac{\alpha_{\nu} \alpha_{t}}{E_{C M}^{2}} \approx \frac{\alpha_{\nu} \alpha_{t}}{M_{N} T},
$$

where $\alpha_{\nu, t}=\lambda_{\nu, t}^{2} / 4 \pi, \lambda_{t}$ is the top quark Yukawa coupling and $E_{C M}^{2}=m_{N} E_{T}$ is the center of mass energy of the scattering particles, where $E_{T} \approx T$ is the energy of the thermal background particle. The scattering rate from relativistic top quarks/squarks is then $\Gamma_{\mathrm{sc}}=n \sigma_{\mathrm{sc}} v=$ $n \sigma_{\mathrm{sc}}$ where $n \approx g_{t} T^{3} / \pi^{2}$ is the number density of top quarks and squarks $\left(g_{t} \approx 10\right.$ is the number of degrees of freedom in top quarks and squarks including spin and color) and $v=1$ for relativistic thermal particles. Thus

$$
\Gamma_{\mathrm{sc}}=\frac{g_{t} \alpha_{\nu} \alpha_{t}}{\pi^{2}} \frac{T^{2}}{M_{N}} .
$$

The important feature of this expression is that the scattering rate is proportional to $T^{2}$, as is $H$ during radiation domination. Therefore the condition for the condensate to evade thermalization by scattering during radiation domination, $\Gamma_{\mathrm{sc}}<H \equiv k_{T} T^{2} / M$, is independent of the temperature,

$$
\lambda_{\nu}^{2} \lessgtr \frac{4 \pi^{3} k_{T}}{g_{t} \alpha_{t}} \frac{M_{N}}{M} .
$$

Using the relation between the Yukawa coupling $\lambda_{\nu}$ and the lightest neutrino mass $m_{\nu_{1}}$, Eq. (7), then implies that

$$
m_{\nu_{1}} \lesssim \frac{4 \pi^{3} k_{T}}{g_{t} \alpha_{t}} \frac{v^{2}}{M} \approx 2 \times 10^{-3}\left(\frac{10}{g_{t}}\right)\left(\frac{0.1}{\alpha_{t}}\right)\left(\frac{v}{100 \mathrm{GeV}}\right)^{2} \mathrm{eV},
$$

where we have used $k_{T}=4.7$. From this we conclude that for $m_{\nu_{1}} \leqslant 10^{-3} \mathrm{eV}$ it should be possible for the Majorana RH sneutrino curvaton to evade thermalization during the radiation dominated era, $T \leq T_{R}$.

This condition is also sufficient to ensure that the curvaton is not thermalized by the background radiation coming from inflaton decays during the IMD era. To see this, note that the expansion rate is related to the back- 
ground radiation temperature during IMD by $T=$ $k_{r}\left(M_{P l} H T_{R}^{2}\right)^{1 / 4}$ where $k_{r}=\left[9 / 5 \pi^{3} g(T)\right]^{1 / 8} \quad$ [26]. Thus $H \propto T^{4}$ while $\Gamma_{\text {sc }}$ is proportional to $T^{2}$ as before. Therefore as $H$ and $T$ increase, the scattering rate becomes small relative to the expansion rate. Thus the largest scattering rate relative to the expansion rate will occur at the lowest temperature during IMD, $T_{R}$. However, we know that $\Gamma_{\mathrm{sc}}<H$ at $T_{R}$ if Eq. (26) is satisfied. Therefore if Eq. (26) is satisfied, the RH sneutrino condensate will evade thermalization during both the radiation dominated and IMD era.

A second thermal effect is the effective mass term generated by the interaction of the $\mathrm{RH}$ sneutrinos with the thermal background MSSM fields [27]. This gives rise to a mass squared term of the form $\lambda_{\nu}^{2}\left\langle\phi^{2}\right\rangle \approx \lambda_{\nu}^{2} T^{2}$, where $\phi$ is a $H_{u}$ or $L_{i}$ field in the thermal background and $\langle\ldots\rangle$ denotes the thermal average. Requiring that this is small compared with $M_{N}^{2}$ implies that $\lambda_{\nu} T_{\max }<M_{N}$, where $T_{\max }$ is the largest background temperature experienced by the coherently oscillating RH sneutrino. This corresponds to the temperature at the onset of RH sneutrino oscillations, $T_{\max }=k_{r}\left(M_{P l} H_{\mathrm{osc}} T_{R}^{2}\right)^{1 / 4} \approx$ $k_{r}\left(M_{P l} m_{N} T_{R}^{2}\right)^{1 / 4}$, where $k_{r} \approx 0.4$ for $g(T)=200$. Therefore

$$
\begin{aligned}
\lambda_{\nu}<\frac{M_{N}^{3 / 4}}{k_{r} T_{R}^{1 / 2} M_{P l}^{1 / 4}}= & 1.4 \times 10^{-7}\left(\frac{M_{N}}{100 \mathrm{GeV}}\right)^{3 / 4} \\
& \times\left(\frac{10^{8} \mathrm{GeV}}{T_{R}}\right)^{1 / 2} .
\end{aligned}
$$

This translates into an upper bound on the lightest neutrino mass

$$
\begin{aligned}
m_{\nu_{1}} \leqslant & 2 \times 10^{-3}\left(\frac{v}{100 \mathrm{GeV}}\right)^{2}\left(\frac{M_{N}}{100 \mathrm{GeV}}\right)^{1 / 2} \\
& \times\left(\frac{10^{8} \mathrm{GeV}}{T_{R}}\right) \mathrm{eV} .
\end{aligned}
$$

Thus $m_{\nu_{1}} \lesssim 10^{-3} \mathrm{eV}$ both allows the Majorana $\mathrm{RH}$ sneutrino condensate to evade thermalization and ensures that thermal effective masses will play no role in the evolution of the RH sneutrino curvaton. The existence of a very light neutrino eigenstate may be regarded as a prediction of the Majorana RH sneutrino curvaton model.

\section{LEPTON ASYMMETRY CONSTRAINTS}

If the RH sneutrino condensate decays at $T_{d \sim}^{>} T_{e w}$ then we must ensure that any lepton asymmetry produced by the decay of the condensate, which would be converted into a baryon asymmetry of equal order of magnitude by sphaleron processes $\left(n_{B} / s=-n_{\text {Linitial }} / 2 s\right.$ [26] $)$, is not larger than the observed baryon asymmetry of the Universe.

In general, a coherently oscillating complex scalar field in SUSY will acquire a global charge asymmetry via the
Affleck-Dine mechanism [22]. Soft SUSY breaking Aand B-terms in the scalar potential [17] will cause the real and imaginary parts of the complex scalar field to oscillate out of phase, such that the scalar field describes an ellipse in the complex plane corresponding to a globally charged scalar field condensate $[10,22]$. However, it is not possible to generate the baryon asymmetry via AD baryogenesis or leptogenesis from the decay of a curvaton, since in this case there would be large baryon isocurvature perturbations due to the fact that in the $\mathrm{AD}$ mechanism both the real and imaginary parts of the curvaton field act to determine the baryon or lepton asymmetry [31].

The global U(1) symmetry in the case of a RH sneutrino condensate is lepton number $\mathrm{L}$ while the $\mathrm{B}$-terms driving the Affleck-Dine mechanism are those associated with the RH neutrino mass terms in Eq. (1). The B-term in Eq. (3) splits the masses of the real and imaginary parts of the RH sneutrino field. For $H^{2} \ll M_{N}^{2}$ the scalar potential is given by

$$
V(N)=\left(M_{N}^{2}+B M_{N}\right) \frac{N_{1}^{2}}{2}+\left(M_{N}^{2}-B M_{N}\right) \frac{N_{2}^{2}}{2},
$$

where $N=\left(N_{1}+i N_{2}\right) / \sqrt{2}$ and we have taken the RH sneutrino mass $m_{N}$ in Eq. (3) to be equal to $M_{N}$. (We may choose the phase of $N$ such that $B$ is real and positive.) The solutions for coherent oscillations of the real and imaginary parts of the RH sneutrino field are then $N_{1}=A_{1} \cos \left(m_{1} t\right)$ and $N_{2}=A_{2} \cos \left(m_{2} t+\delta\right)$, where $m_{1}=\left(M_{N}^{2}+B M_{N}\right)^{1 / 2}, m_{2}=\left(M_{N}^{2}-B M_{N}\right)^{1 / 2}$ and $A_{1,2} \propto$ $a^{-3 / 2}$. We have included a phase shift $\delta$, since this would be generally expected to arise during the initial transition from Hubble damped evolution to coherent oscillations. We will see that $\delta$ has a negligible effect on the net lepton asymmetry. Since the B-term in the scalar field equation for $N$ becomes dynamically significant, $B M_{N} \gtrsim H^{2}$, only once $H \approx M_{N}$, corresponding to the onset of coherent oscillations, there is no reason to expect $N$ to be along the real direction at the onset of RH sneutrino oscillations. Thus we expect the phase of $N$ initially to be random and of the order of 1 , such that $A_{1}$ and $A_{2}$ will be of the same order of magnitude.

The lepton asymmetry density in the RH sneutrino condensate, $n_{L \text { cond }}$, is given by

$$
n_{\text {Lcond }}=i\left(\dot{N}^{\dagger} N-N^{\dagger} \dot{N}\right)=\left(\dot{N}_{2} N_{1}-\dot{N}_{1} N_{2}\right) .
$$

Therefore

$$
\begin{aligned}
n_{L \text { cond }}= & A_{1} A_{2}\left[m_{1} \sin \left(m_{1} t\right) \cos \left(m_{2} t+\delta\right)\right. \\
& \left.-m_{2} \sin \left(m_{2} t+\delta\right) \cos \left(m_{1} t\right)\right] .
\end{aligned}
$$

For example, for the case $\delta=0$, if we consider $M_{N} \gg B$ then $m_{1} \approx M_{N}+\delta m / 2, m_{2} \approx M_{N}-\delta m / 2$, where $\delta m \approx$ $B$. Then 


$$
n_{L \mathrm{cond}} \approx A_{1} A_{2} m\left[\sin (\delta m t)+\frac{\delta m}{2 m} \sin (2 m t)\right] .
$$

Thus, for $m, \delta m \gg H\left(T_{d}\right)$, there will be rapid oscillations of the lepton asymmetry between large positive and negative lepton number over the time scale of condensate decay, $\Gamma_{d}^{-1} \approx H^{-1}\left(T_{d}\right)$.

We next calculate the net lepton asymmetry produced by the decay of the RH sneutrino condensate. Although the lepton asymmetry in the condensate is timedependent, once the lepton number is transferred to the thermal background it will be conserved. (We neglect the effect of sphaleron processing for now, which will not affect the magnitude of the lepton asymmetry.) This follows since lepton number violating scattering processes due to $\mathrm{RH}$ neutrino exchange are out of equilibrium at temperatures below $T_{f} \approx 1.2 \times$ $10^{11}\left(1 \mathrm{eV} / m_{\nu}\right)^{2} \mathrm{GeV}$ [32]. Therefore the rate of increase of the thermal background lepton number density, $n_{L}$, from $\mathrm{RH}$ sneutrino condensate decay is given by

$$
\frac{1}{a^{3}} \frac{d\left(a^{3} n_{L}\right)}{d t}=\Gamma_{d} e^{-\Gamma_{d} t} n_{L \mathrm{cond}},
$$

where we have included a factor $e^{-\Gamma_{d} t}$ in order to account for the decay of the RH sneutrino condensate and the scale factor terms account for the expansion of the Universe. Thus

$$
\begin{aligned}
n_{L}= & \Gamma_{d} A_{1} A_{2} \int_{0}^{\infty} e^{-\Gamma_{d} t}\left\{\left[m_{1} \sin \left(m_{1} t\right) \cos \left(m_{2} t\right)\right.\right. \\
& \left.-m_{2} \sin \left(m_{2} t\right) \cos \left(m_{1} t\right)\right] \cos \delta-\left[m_{1} \sin \left(m_{1} t\right)\right. \\
& \left.\left.\times \sin \left(m_{2} t\right)+m_{2} \cos \left(m_{1} t\right) \cos \left(m_{2} t\right)\right] \sin \delta\right\} d t .
\end{aligned}
$$

The integrals may be calculated exactly, with the result

$$
\begin{aligned}
n_{L}= & \frac{\Gamma_{d} A_{1} A_{2}\left(m_{1}^{2}-m_{2}^{2}\right)\left[\Gamma_{d}^{2}+m_{1}^{2}+m_{2}^{2}\right] \cos \delta}{\left[\Gamma_{d}^{2}+\left(m_{1}-m_{2}\right)^{2}\right]\left[\Gamma_{d}^{2}+\left(m_{1}+m_{2}\right)^{2}\right]} \\
& -\frac{\Gamma_{d}^{2} A_{1} A_{2}\left(m_{2} \Gamma_{d}^{2}+3 m_{1}^{2} m_{2}+m_{2}^{3}\right) \sin \delta}{\left[\Gamma_{d}^{2}+\left(m_{1}-m_{2}\right)^{2}\right]\left[\Gamma_{d}^{2}+\left(m_{1}+m_{2}\right)^{2}\right]} .
\end{aligned}
$$

For small $\Gamma_{d}^{2}$ compared with $\left(m_{1}-m_{2}\right)^{2}$, and assuming that the natural value of $\delta$ is not very close to $\pi / 2$, the term proportional to $\sin \delta$ is negligible and Eq. (35) becomes

$$
n_{L} \approx \frac{\Gamma_{d} A_{1} A_{2}\left(m_{1}^{2}+m_{2}^{2}\right) \cos \delta}{\left(m_{1}^{2}-m_{2}^{2}\right)}=\frac{\Gamma_{d} A_{1} A_{2} M_{N} \cos \delta}{B} .
$$

We wish to express this in the form $n_{L} / s$. The energy density in the RH sneutrino condensate is given by

$$
\rho=\rho_{1}+\rho_{2}=\frac{m_{1}^{2} A_{1}^{2}}{2}+\frac{m_{2}^{2} A_{2}^{2}}{2} .
$$

The entropy density at $T_{d}$ is related to $\rho$ by $s=4 \rho / 3 T_{d}$ [26], where we assume the radiation energy density from RH sneutrino decay at $T_{d}$ equals $\rho$. Thus the lepton number to entropy density is given by

$$
\frac{n_{L}}{s}=\frac{3 T_{d} \Gamma_{d} M_{N} \cos \delta}{2 B} \frac{A_{1} A_{2}}{\left(m_{1}^{2} A_{1}^{2}+m_{2}^{2} A_{2}^{2}\right)} .
$$

With $A_{1} \sim A_{2}, m_{1}^{2} \approx m_{2}^{2} \approx M_{N}^{2}, \cos \delta \approx 1$ and $B \lesssim M_{N}$, this becomes

$$
\frac{n_{L}}{s} \approx \frac{T_{d} \Gamma_{d}}{M_{N} B} .
$$

Using $\Gamma_{d}=k_{T} T_{d}^{2} / M$, the lepton asymmetry from B-term $\mathrm{AD}$ leptogenesis is therefore given by

$$
\frac{n_{L}}{s} \approx \frac{k_{T} T_{d}^{3}}{B M_{N} M} .
$$

Requiring that $n_{L} / s \lessgtr 10^{-10}$ imposes the constraint

$$
T_{d} \lesssim 7.9 \times 10^{3}\left(\frac{B}{100 \mathrm{GeV}}\right)^{1 / 3}\left(\frac{M_{N}}{100 \mathrm{GeV}}\right)^{1 / 3} \mathrm{GeV}
$$

With $T_{d}$ given by Eq. (9), this implies an upper bound on $M_{N}$,

$$
M_{N} \lesssim 1.3 \times 10^{5}\left(\frac{10^{-4} \mathrm{eV}}{m_{\nu_{1}}}\right)^{3 / 4}\left(\frac{B}{100 \mathrm{GeV}}\right)^{1 / 2} \mathrm{GeV} .
$$

This may also be expressed as an upper bound on $T_{d}$,

$$
T_{d} \lesssim 8.6 \times 10^{4}\left(\frac{10^{-4} \mathrm{eV}}{m_{\nu_{1}}}\right)^{1 / 4}\left(\frac{B}{100 \mathrm{GeV}}\right)^{1 / 2} \mathrm{GeV}
$$

Thus if $m_{\nu_{1}}$ is not very much smaller than the mass of the other neutrino eigenstates then the lepton asymmetry constraint Eq. (42) favors a relatively light RH neutrino mass, $m_{N} \lesssim 10^{5} \mathrm{GeV}$, while Eq. (43) implies that the decay temperature is relatively low, $T_{d} \lesssim 10^{5} \mathrm{GeV}$.

The analysis of the B-term AD mechanism given above is similar to that recently given in [21] in the more general context of AD leptogenesis from a RH sneutrino condensate. Some of the analytical results we have obtained here should be useful in this more general context, in particular, the expression for the lepton asymmetry resulting from decay of the condensate, Eq. (35). From Eq. (35) we see that the maximum asymmetry will be obtained for $\Gamma_{d} \approx\left|m_{1}-m_{2}\right| \approx B$, in agreement with [21]. However, it is worth emphasizing that a significant asymmetry can still be obtained even if $\Gamma_{d} \ll B$, as is clear from Eq. (39).

There are two obvious possibilities for the origin of the baryon asymmetry in the Majorana RH sneutrino curvaton model: electroweak baryogenesis [23] or RH sneutrino leptogenesis based on conventional leptogenesis via $C P$-violating decays of the condensate $\mathrm{RH}$ sneutrinos [13]. Both of these require that the curvaton condensate decays before the electroweak phase transition. In the case where the energy density is dominated by the $\mathrm{RH}$ sneutrino condensate, it was shown in [13] that the lepton asymmetry from CP-violating decays of the condensate $\mathrm{RH}$ sneutrinos is given by 


$$
\frac{n_{L}}{s} \approx 0.7 \times 10^{-10}\left(\frac{T_{d}}{10^{6} \mathrm{GeV}}\right)\left(\frac{m_{\nu_{3}}}{0.05 \mathrm{eV}}\right) \delta_{\mathrm{eff}}
$$

where $\delta_{\text {eff }} \lesssim 1$ is the $C P$-violating phase. Therefore $\mathrm{RH}$ sneutrino leptogenesis requires that $T_{d} \approx 10^{6} \mathrm{GeV}$ if $\delta_{\text {eff }} \approx 1$. From the lepton asymmetry constraint, Eq. (41), this would require that $M_{N} \geqslant 10^{8} \mathrm{GeV}$. Equation (42) in turn requires that $m_{\nu_{1}} \lesssim 10^{-8} \mathrm{eV}$. Therefore RH sneutrino leptogenesis is only possible if the lightest neutrino mass is extremely small compared with the mass scale suggested by solar and atmospheric neutrino oscillations.

\section{CONSEQUENCES OF THE CONSTRAINTS}

We next consider the consequences of the simultaneous application of the constraints derived above for the parameter space of the Majorana RH sneutrino curvaton model. We will consider the allowed region of the $\left(M_{N}, T_{R}\right)$ parameter space for different values of $m_{\nu_{1}}$ satisfying the thermalization upper bound $m_{\nu_{1}} \lesssim$ $10^{-3} \mathrm{eV}$ and for various lower bounds on $T_{d}$.

The constraints we apply are: (i) the upper bound on $M_{N}$ from curvaton dominance combined with $N_{\text {osc }} \lesssim M$, Eq. (12), (ii) the lower bound on $M_{N}$ from requiring that the RH sneutrino decay temperature in Eq. (9) is greater than a given value and (iii) the upper bound on $M_{N}$ from the AD leptogenesis constraint, Eq. (42). In addition, we will consider the upper bound on $M_{N}$ for which a RH sneutrino curvaton is compatible with a solution of the Dterm inflation cosmic string problem for the case $c=1$, Eq. (21). (We consider $g=1$ for the $U(1)_{F I}$ gauge coupling throughout.)

In Fig. 1 we show the constraints for the case $m_{\nu_{1}}=$ $10^{-3} \mathrm{eV}$ (the largest value consistent with thermalization) and lower bound on $T_{d}$ equal to $100 \mathrm{GeV}$, corresponding to the value for which electroweak baryogenesis is possible. The case $m_{\nu_{1}} \approx 10^{-3} \mathrm{eV}$ might be expected if $m_{\nu_{1}}$ is not very much smaller than $m_{\nu_{2,3}} \sim$ $0.01-0.1 \mathrm{eV}$. From Fig. 1 the allowed range of $M_{N}$ is constrained to be between $47 \mathrm{GeV}$ and $2.3 \times 10^{4} \mathrm{GeV}$. The allowed range of $M_{N}$ becomes narrower for $T_{R}<$ $10^{6} \mathrm{GeV}$, where the curvaton dominance constraint becomes more important than the AD leptogenesis constraint. $T_{R}$ cannot be smaller than $2.7 \mathrm{TeV}$. It is interesting and perhaps significant that the allowed range of $M_{N}$ contains the $100 \mathrm{GeV}-1 \mathrm{TeV}$ mass scale, a natural mass scale of particle physics models.

In Fig. 2 we consider a more restrictive possibility with $m_{\nu_{1}}=10^{-4} \mathrm{eV}$ and $T_{d}>1 \mathrm{TeV}$. The allowed range of $M_{N}$ is significantly narrower in this case compared with Fig. 1, with $M_{N}$ between $1.4 \times 10^{3} \mathrm{GeV}$ and $1.3 \times$ $10^{5} \mathrm{GeV}$. In general, increasing the lower bound on $T_{d}$ increases the lower bound on $M_{N}\left(\propto T_{d}\right.$, Eq. (9)) while having no effect on the upper bound. Decreasing $m_{\nu_{1}}$

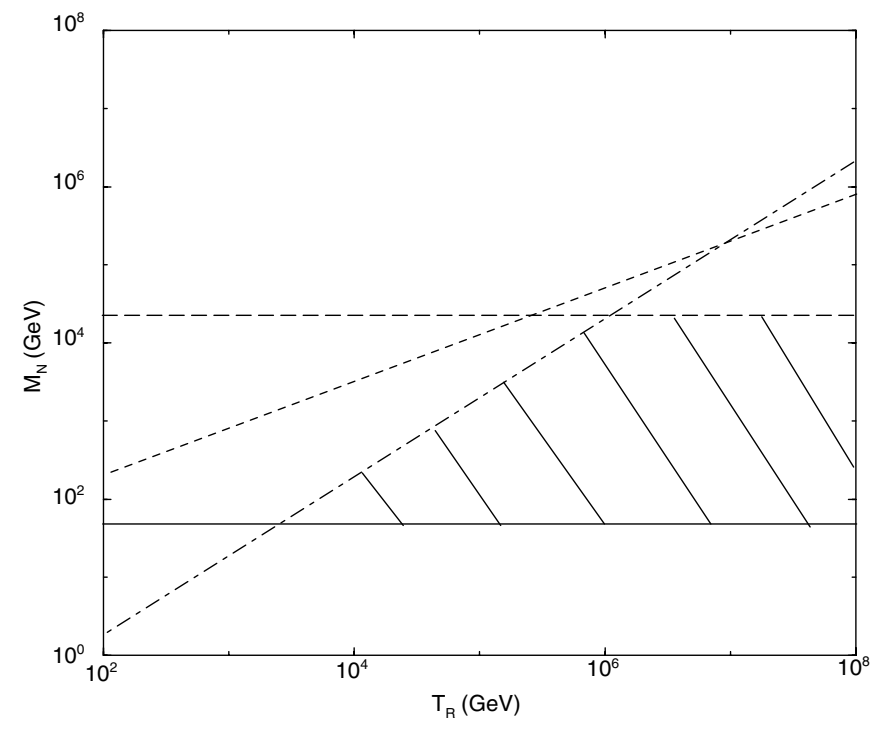

FIG. 1. Right-handed neutrino mass vs reheating temperature for $m_{\nu_{1}}=10^{-3} \mathrm{eV}$ and $T_{d}>10^{2} \mathrm{GeV}$. The solid line is the lower bound on $M_{N}$ from $T_{d}>10^{2} \mathrm{GeV}$. The long-dashed line is the upper bound from B-term AD leptogenesis. The dotdashed line is the upper bound from curvaton dominance. These define the allowed region, shown hatched. The shortdashed line is the D-term inflation upper bound on $M_{N}$ for $c=1$.

raises both the upper bound $\left(\propto m_{\nu_{1}}^{-3 / 4}\right.$, Eq. (42)) and, to a lesser extent, the lower bound $\left(\propto m_{\nu_{1}}^{-1 / 2}\right.$ Eq. (9)).

In Fig. 3 we show the case where the lower bound on $T_{d}$ is $10^{6} \mathrm{GeV}$, compatible with RH sneutrino leptogenesis. In this case the lower bound on $M_{N}$ is $5.0 \times 10^{8} \mathrm{GeV}$. We have shown the allowed parameter space for the case where $m_{\nu_{1}}=10^{-9} \mathrm{eV}$, which is just compatible with RH sneutrino leptogenesis, as illustrated by the very

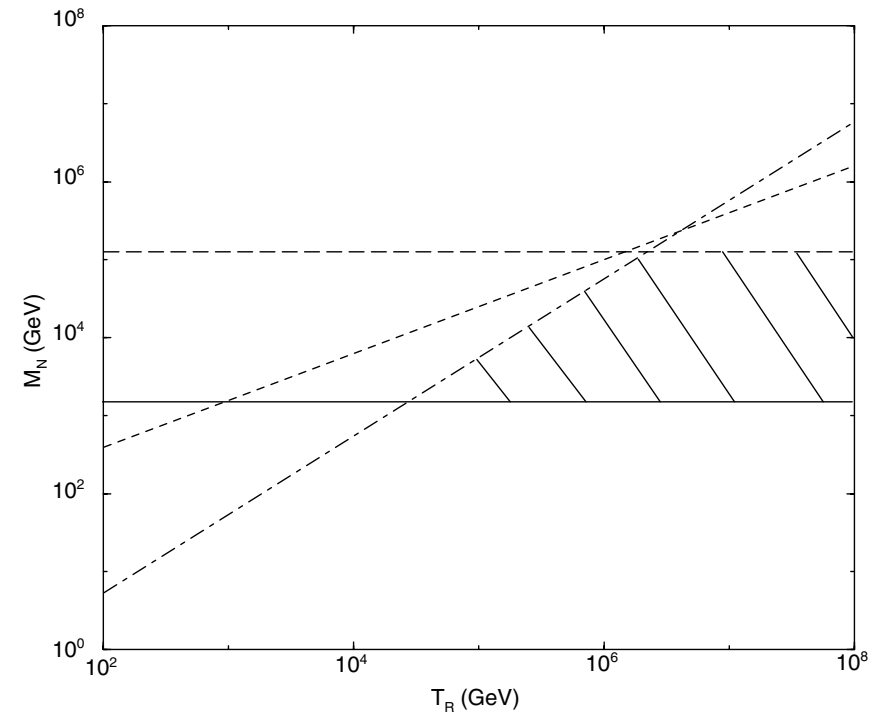

FIG. 2. Right-handed neutrino mass vs reheating temperature for $m_{\nu_{1}}=10^{-4} \mathrm{eV}$ and $T_{d}>10^{3} \mathrm{GeV}$. 


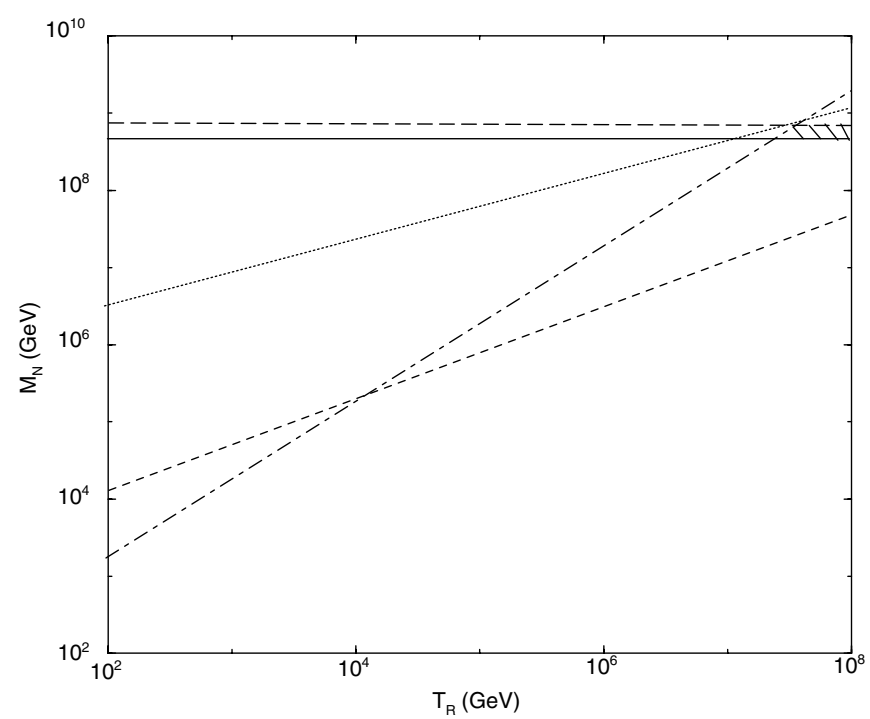

FIG. 3. Right-handed neutrino mass vs reheating temperature for $m_{\nu_{1}}=10^{-9} \mathrm{eV}$ and $T_{d}>10^{6} \mathrm{GeV}$. The D-term inflation upper bound for the case $c=2.5$ is also shown (dotted line).

small region of the parameter space compatible with the constraints. For $m_{\nu_{1}} \gtrsim 10^{-8} \mathrm{eV} \mathrm{RH}$ sneutrino leptogenesis is ruled out by the AD leptogenesis constraint. The case $T_{d}=10^{6} \mathrm{GeV}$ is not compatible with a curvaton solution of the D-term inflation cosmic string problem when $c=1$. However, larger values of $c$ (but still of the order of 1) will allow a solution, as shown by the upper bound for the case $c=2.5$.

We conclude that unless $m_{\nu_{1}}$ is very small compared with the scale of neutrino masses responsible for solar and atmospheric neutrino oscillations, the Majorana RH sneutrino curvaton favors relatively low values of $M_{N}$ and $T_{d}$. The region of the parameter space compatible with a Majorana RH sneutrino curvaton is generally consistent with a curvaton solution of the D-term inflation cosmic string problem. RH sneutrino leptogenesis is ruled out as a source of the baryon asymmetry unless $m_{\nu_{1}} \lesssim$ $10^{-8} \mathrm{eV}$, thus favoring electroweak baryogenesis as the origin of the baryon asymmetry. However, it may be significant that RH sneutrino leptogenesis is not entirely ruled out as a source of the baryon asymmetry.

\section{CONCLUSIONS}

We have considered the conditions under which a Majorana RH sneutrino could serve as a curvaton. One condition is the absence of Planck-scale suppressed corrections to the RH sneutrino scalar potential to a high order. As the nature of such corrections requires speculation about the nature of the underlying full theory, we have considered as one possibility the case where such corrections are absent. This might be explained by an Rsymmetry, although this requires an additional suppres- sion of interaction terms between the observable sector and SUSY breaking hidden sector superfields in the superpotential. A second condition is that the RH sneutrino condensate is not thermalized by scattering from the thermal background particles. This requires a model of neutrino masses in which one of the RH neutrino mass eigenstates has significantly weaker Yukawa couplings to the MSSM fields than the other RH neutrinos. The lightest neutrino mass eigenstate must then satisfy $m_{\nu_{1}} \lesssim$ $10^{-3} \mathrm{eV}$, which may be regarded as a prediction of the scenario. The Majorana RH sneutrino curvaton scenario can therefore be ruled out experimentally by the observation of a degenerate neutrino mass spectrum, $m_{\nu_{1}} \approx m_{\nu_{2}} \approx m_{\nu_{3}}$.

We have shown that a lepton asymmetry will be induced in the RH sneutrino condensate via the AD mechanism associated with SUSY breaking B-terms. Requiring that the baryon asymmetry coming from sphaleron conversion of this lepton asymmetry is smaller than the observed baryon asymmetry imposes an upper bound on $M_{N}$. Unless $m_{\nu_{1}}$ is very small compared with the mass scale of solar and atmospheric neutrino oscillations, relatively small values of $M_{N}\left(\lesssim 10^{5} \mathrm{GeV}\right)$ are required for consistency with the AD leptogenesis constraint. Some of the analytical results we have obtained here should be useful in the more general analysis of AD leptogenesis from a RH sneutrino condensate.

The AD leptogenesis constraint, combined with a lower bound on the RH sneutrino condensate decay temperature and the requirement that the RH sneutrino curvaton condensate dominates the energy density before it decays, restricts the region of the $\left(M_{N}, T_{R}\right)$ parameter space consistent with a $\mathrm{RH}$ sneutrino curvaton. The range of $M_{N}$ consistent with a RH sneutrino curvaton is typically smaller than that considered in the conventional seesaw mechanism where $\lambda_{\nu}$ is assumed to be of the order of the charged lepton Yukawa couplings, which requires $M_{N} \approx 10^{9} \mathrm{GeV}$. However, the allowed range of $M_{N}$ for $m_{\nu_{1}} \approx 10^{-3} \mathrm{eV}$ and $T_{d} \geqslant 100 \mathrm{GeV}$ is consistent with the $100 \mathrm{GeV}-1 \mathrm{TeV}$ mass scale, a natural mass scale in particle physics.

An important motivation for a SUSY curvaton is Dterm hybrid inflation. We have shown that the region of the parameter space allowed by the constraints on the Majorana RH sneutrino curvaton is generally consistent with a solution of the D-term inflation cosmic string problem. D-term inflation naturally accounts for the absence of order $\mathrm{H}^{2}$ corrections during inflation in both the inflaton and curvaton scalar potentials. Therefore a combined D-term inflation/Majorana RH sneutrino curvaton model could provide a completely consistent model of SUSY inflation.

The only obvious candidates for the origin of the baryon asymmetry in the Majorana RH sneutrino curvaton scenario are electroweak baryogenesis and RH sneu- 
trino leptogenesis, $\mathrm{AD}$ leptogenesis being generally ruled out for a curvaton [31]. We find that RH sneutrino leptogenesis via $C P$-violating decays of the $\mathrm{RH}$ sneutrinos is consistent with the Majorana RH sneutrino curvaton only if the lightest neutrino mass eigenstate satisfies $m_{\nu_{1}} \lesssim 10^{-8} \mathrm{eV}$. Therefore RH sneutrino leptogenesis appears to be disfavored, although it may be significant that it is not completely ruled out. This suggests that electroweak baryogenesis is the most likely source of the baryon asymmetry in this scenario.
[1] S. Mollerach, Phys. Rev. D 42, 313 (1990); A. Linde and V. Mukhanov, Phys. Rev. D 56, 535 (1997); K. Enqvist and M. S. Sloth, Nucl. Phys. B626, 395 (2002).

[2] T. Moroi and T. Takahashi, Phys. Lett. B 522, 215 (2001); Phys. Rev. D 66, 063501 (2002).

[3] D. H. Lyth and D. Wands, Phys. Lett. B 524, 5 (2002); D. Lyth, C. Ungarelli, and D. Wands, Phys. Rev. D 67, 023503 (2003).

[4] N. Bartolo and A. R. Liddle, Phys. Rev. D 65, 121301 (2002); K. Dimopoulos and D. H. Lyth, Phys. Rev. D 69, 123509, (2004); M. Bastero-Gil, V. Di Clemente, and S. F. King, Phys. Rev. D 67, 103516 (2003); K. Enqvist, S. Kasuya, and A. Mazumdar, Phys. Rev. Lett. 90, 091302 (2003); K. Dimopoulos, D. H. Lyth, A. Notari, and A. Riotto, J. High Energy Phys. 07 (2003) 053; D. H. Lyth and D. Wands, Phys. Rev. D 68, 103516 (2003); K. Dimopoulos, G. Lazarides, D. H. Lyth, and R. Ruiz de Austri, Phys. Rev. D 68, 123515 (2003); K. Dimopoulos, G. Lazarides, D. H. Lyth, and R. Ruiz de Austri, J. High Energy Phys. 05 (2003) 057; M. Postma and A. Mazumdar, J. Cosmol. Astropart. Phys. 01 (2004) 005; S. Kasuya, M. Kawasaki, and F. Takahashi, Phys. Lett. B 578, 259 (2004); M. Bastero-Gil, V. Di Clemente, and S. F. King, Phys. Rev. D 70, 023501 (2004); C. Gordon and K. A. Malik, Phys. Rev. D 69, 063508 (2004); D. H. Lyth, Phys. Lett. B 579, 239 (2004).

[5] C. Gordon and A. Lewis, Phys. Rev. D 67, 123513 (2003).

[6] M. Postma, Phys. Rev. D 67, 063518 (2003).

[7] J. McDonald, Phys. Rev. D 68, 043505 (2003).

[8] K. Enqvist, A. Jokinen, S. Kasuya, and A. Mazumdar, Phys. Rev. D 68, 103507 (2003); K. Enqvist, Mod. Phys. Lett. A 19, 1421 (2004).

[9] J. McDonald, J. Cosmol. Astropart. Phys. 12 (2003) 005 .

[10] J. McDonald, Phys. Rev. D 69, 103511 (2004).

[11] E. Halyo, Phys. Lett. B 387, 43 (1996); P. Binetruy and G. Dvali, Phys. Lett. B 388, 241 (1996).

[12] M. Endo, M. Kawasaki, and T. Moroi, Phys. Lett. B 569, 73 (2003).

[13] K. Hamaguchi, H. Murayama, and T. Yanagida, Phys. Rev. D 65, 043512 (2002).

[14] M. Gell-Mann, P. Ramond, and R. Slansky, Supergravity (North-Holland, Amsterdam, 1979); T. Yanagida, in Proc. of the Workshop on Unified Theory and Baryon Number of the Universe (KEK, Japan, 1979); R. N.
Mohnpatra and G. Senjanovic, Phys. Rev. Lett. 44, 912 (1980).

[15] R. N. Mohapatra, hep-ph/0402035.

[16] S. F. King, Phys. Lett. B 439, 350 (1998); Nucl. Phys. B562, 57 (1999); Nucl. Phys. B576, 85 (2000); Nucl. Phys. B, Proc. Suppl. 95, 261 (2001).

[17] H. P. Nilles, Phys. Rep. 110, 1 (1984).

[18] G. F. Giudice and A. Masiero, Phys. Lett. B 206, 480 (1988).

[19] R. Holman, S. Hsu, T. Kephart, E. Kolb, R. Watkins, and L. Widrow, Phys. Lett. B 282, 132 (1992); M. Kamionkowski and J. March-Russell, Phys. Lett. B 282, 137 (1992); S. M. Barr and D. Seckel, Phys. Rev. D 46, 539 (1992); S. M. Lusignoli and M. Roncadelli, Phys. Lett. B 283, 278 (1992).

[20] R. Kallosh, A. Linde, D. Linde, and L. Susskind, Phys. Rev. D 52, 912 (1995).

[21] R. Allahverdi and M. Drees, Phys. Rev. D 69, 103522 (2004).

[22] I. A. Affleck and M. Dine, Nucl. Phys. B249, 361 (1985).

[23] A. Riotto and M. Trodden, Annu. Rev. Nucl. Part. Sci. 49, 35 (1999).

[24] J. R. Ellis, J. E. Kim, and D. V. Nanopoulos, Phys. Lett. B145, 181 (1984); J. R. Ellis, D. V. Nanopoulos, and S. Sarkar, Nucl. Phys. B259, 175 (1985); S. Sarkar, Rep. Prog. Phys. 50, 1493 (1996).

[25] M. Yu. Khlopov and A. D. Linde, Phys. Lett. B138, 965 (1984).

[26] E.W. Kolb and M.S. Turner, The Early Universe (Addison-Wesley, Reading, MA, 1990).

[27] R. Allahverdi, B. A. Campbell, and J. R. Ellis, Nucl. Phys. B579, 355 (2000).

[28] M. Dine, W. Fischler, and D. Nemeschansky, Phys. Lett. B136, 169 (1984); G. D. Coughlan, R. Holman, P. Ramond, and G. G. Ross, Phys. Lett. B140, 44 (1984); O. Bertolami and G. G. Ross, Phys. Lett. B 183, 163 (1987); G. Dvali, Phys. Lett. B 355, 78 (1995).

[29] E. J. Copeland, A. R. Liddle, D. H .Lyth, E. D. Stewart, and D. Wands, Phys. Rev. D 49, 6410 (1994).

[30] M. Dine, L. Randall, and S. Thomas, Phys. Rev. Lett. 75, 398 (1995); Nucl. Phys. B458, 291 (1996).

[31] K. Hamaguchi, M. Kawasaki, T. Moroi, and F. Takahashi, Phys. Rev. D 69, 063504 (2004).

[32] J. M. Cline, K. Kainulainen, and K. A. Olive, Phys. Rev. Lett. 71, 2372 (1993). 